\title{
Cross-Phase Modulation Instability in PM ANDi Fiber-Based Supercontinuum Generation
}

\author{
Etienne Genier ${ }^{1,2, *}$, Amar N. Ghosh ${ }^{2}$, Swetha Bobba ${ }^{2}$, Patrick Bowen ${ }^{1}$, Peter M. \\ Moselund $^{1}$, Ole Bang ${ }^{1,3}$, John M. Dudley ${ }^{2}$, And Thibaut Sylvestre ${ }^{2}$ \\ ${ }^{1}$ NKT Photonics A/S, Blokken 84, DK-3460, Birkerød, Denmark \\ ${ }^{2}$ Institut FEMTO-ST, UMR 6174 CNRS-Université de Franche-Comté, 25030 Besançon, France \\ ${ }^{3}$ DTU Fotonik, Department of Photonics Engineering, Technical University of Denmark, 2800 Kgs. Lyngby, Denmark \\ *Corresponding author: etienne.genier@nktphotonics.com
}

Compiled May 6, 2020

We demonstrate broadband supercontinuum generation in an all-normal dispersion polarizationmaintaining photonic crystal fiber and we report the observation of a cross-phase modulation instability sideband that is generated outside of the supercontinuum bandwidth. We demonstrate this sideband is polarized on the slow axis and can be suppressed by pumping on the fiber's fast axis. We theoretically confirm and model this nonlinear process using phase-matching conditions and numerical simulations, obtaining good agreement with the measured data. () 2020 Optical Society of America

http://dx.doi.org/10.1364/ao.XX.XXXXXX

All-normal dispersion (ANDi) optical fibers have recently emerged as attractive platforms to improve the noise and coherence of supercontinuum (SC) generation beyond the limits of anomalous SC generation [1-4]. ANDi SC generation is based on two fully coherent nonlinear effects: self-phase modulation (SPM) and optical wave breaking (OWB) $[2,5]$ while anomalous SC generation is typically susceptible to or even generated by incoherent nonlinear effects [6]. Despite this, ANDi SC generation has its own limitations, and being both very sensitive to Raman noise $[2-4,7]$ and requiring low and flat fiber dispersion engineering that is technically challenging to achieve [1]. When pumping with femtosecond pulses, it has been shown that other factors should be considered including polarization modulation instability (PMI) or the amplitude noise of the laser, which both can drastically degrade the relative intensity noise (RIN) and coherence $[3,4,8]$. These factors limit the available parameter space for coherent SC generation, however, fs-pumped ANDi SC generation still has significant potential to generate temporally coherent SC with realistic laser parameters, a feature that is unachievable in the anomalous dispersion regime. This gives such systems potential in a range of fields including optical coherence tomography (OCT), optical metrology, photoacoustic imaging, and spectroscopy [9-13].

In this work, we investigate SC generation in a PM-ANDi silica photonic crystal fiber (PCF) with a femtosecond stable optical parametric oscillator (OPO) with intention to suppress
PMI. However, in doing this, we discovered the generation of a sideband outside the SC bandwidth which was not observed in previous PM-ANDi SC generation [14, 15]. We identify this sideband as the result of cross-phase modulation instability (XPMI) process that builds up from coherent SC generation and OWB. As it is described in [16, 17], XPMI is usually observed when a beam is launched at a $45^{\circ}$ angle from the principal axis of a highly birefringent fiber. This beam is then split into two linearly polarized modes on each axis that will nonlinearly interact with each other to generate two frequency-detuned and crosspolarized four-wave mixing (FWM) sidebands [16]. However, this XPMI process has never been observed before through the stimulation of a fs-SC generation but only via spontaneous generation of the interaction of picosecond or nanosecond pulses.

Our results show that we can generate a stimulated XPMI sideband in a PM-ANDi PCF using femtosecond pulses. As expected, this sideband is most powerful while pumping the fiber at $45^{\circ}$ off the axes. We also demonstrate this sideband can be completely suppressed when pumping the fiber on the fast axis. Significantly, we note that while this sideband is observable outside the SC bandwidth with a low power pump, at higher powers the bandwidth of the supercontinuum will cover the sideband and significantly degrade the SC coherence.

The experimental setup used to observe and analyze SC and XPMI generation in the PM-ANDi PCF is shown in Fig. 1. As a pump laser, we used a Ti:Sa femtosecond pulsed laser (Coherent Chameleon) tunable from $680 \mathrm{~nm}-1080 \mathrm{~nm}$, delivering $200 \mathrm{fs}$ pulse duration at a $80 \mathrm{MHz}$ repetition rate with a maximum average power of $450 \mathrm{~mW}$ at $1040 \mathrm{~nm}$. The output power is controlled using a variable neutral density filter (ND). A half-wave plate is used to turn the input polarization state at the fiber input while the polarizer at the output of the fiber is used to observe the spectral content of the light of each axis. A 40x microscope objective is used to couple the light into the $40 \mathrm{~cm}$ of PM ANDi PCF - the NL-1050-NE-PM from NKT Photonics. This fiber has a relative hole size of $\mathrm{d} / \mathrm{L}=0.45$, a small hole-to-hole pitch of $1.44 \mu \mathrm{m}$, and a nonlinear coefficient of $\gamma=26.8 \mathrm{~W}^{-1} \mathrm{~km}^{-1}$ at 1040 $\mathrm{nm}$. A set of 2 aspheric lenses is used to collimate the output beam and then focus it to the multimode pick-up fiber.

The dispersion was measured using white-light interferometry and calculated for an idealized structure with a uniform 


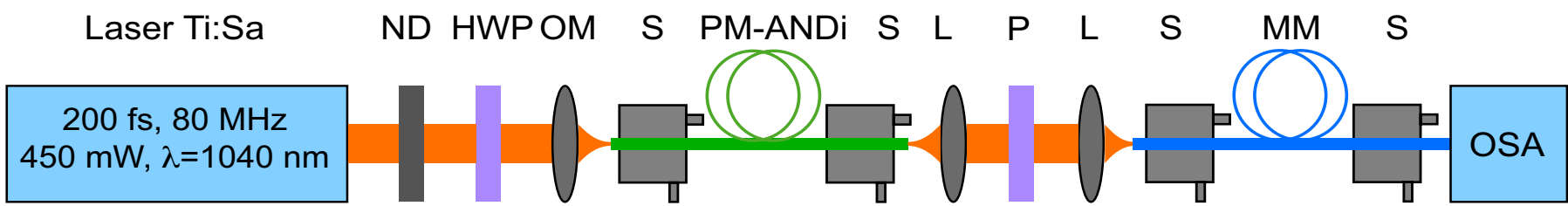

Fig. 1. Schematic of the setup, including a wavelength tunable Ti:Sa femtosecond laser, a variable neutral density filter (ND), a half wave plate (HWP), a 40x microscope objective (OM), aspheric lenses (L), 3D translation stages (S), $40 \mathrm{~cm}$ of all normal dispersion PCF (PM-ANDi), a polarizer (P), 2 m of multimode pick-up fiber (MM), and an optical spectrum analyzer (OSA).

hole structure with fixed pitch and hole diameter using COMSOL. The COMSOL calculation fits the measurements well and is shown as the blue curve in Fig. 2. Numerical modelling with this dispersion did not reproduce the experiment. Because the uncertainty in the measurement increases when the dispersion is weak. We therefore considered the dispersion given by the brown curve, which is shifted upwards in the center as the dispersion approaches zero. This is still within the measurement uncertainties and is able to reproduce the experiments as we will demonstrate. The dispersion of the two axes is very similar thus the broadening expected while pumping on axis should be similar for each polarization. As expected, the small holes of the PCF give a confinement loss edge wavelength significantly below the material loss edge wavelength, calculated to be at $1450 \mathrm{~nm}$ using COMSOL (see Fig. 2, green curve). The dispersion profile has a minimum of $-13 \mathrm{ps} / \mathrm{nm} / \mathrm{km}$ at $1040 \mathrm{~nm}$ and is rather symmetrical within the low-loss window. The polarization-maintaining effect of this fiber is stress-rod induced, with a slight degree of core-ellipticity that causes a linearly increasing birefringence [18], which goes from $2 \cdot 5 \cdot 10^{-4}$ at $850 \mathrm{~nm}$ to $6 \cdot 8 \cdot 10^{-4}$ at $1300 \mathrm{~nm}$, as shown in Fig. 2 (red circles).

We pumped the fiber at $1040 \mathrm{~nm}$, at the minimum dispersion wavelength (MDW), thus we should expect the broadest SC spectrum for a given power and input angle. Figure 3 shows the spectral evolution while pumping on the slow axis, fast axis and at $45^{\circ}$. The broadest SC spectrum (bandwidth at -20 $\mathrm{dB}$ is $460 \mathrm{~nm}$ ) is obtained by pumping on the slow axis while the narrowest is obtained by pumping at $45^{\circ}$. The spectrum generated when pumping on the fast axis is narrower than when

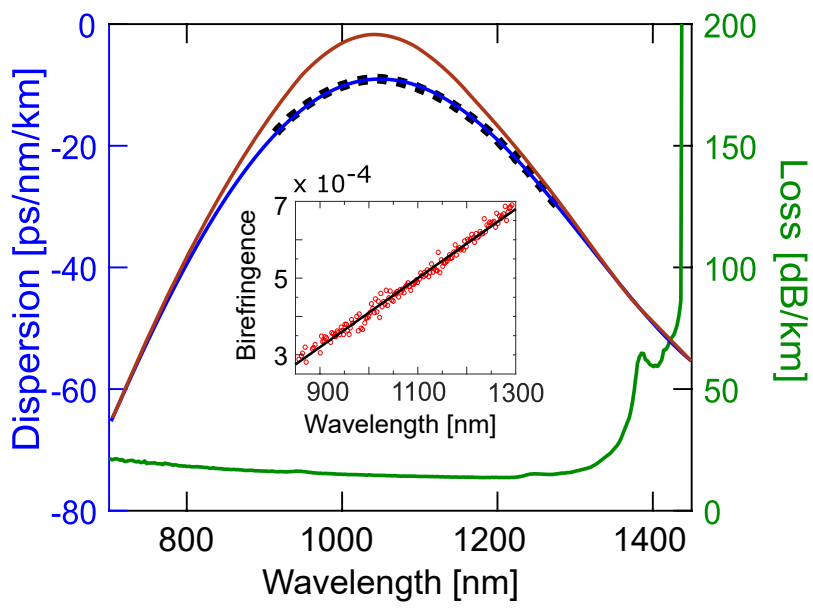

Fig. 2. Comsol (solid blue), experimental (black dots) and modeled (dash brown) dispersion profiles, and fiber losses (solid green) of the NL-1050-NE-PM ANDi PCF. The inset shows the dispersion of the group birefringence: the linear fitting (solid black) and measured data (circles red). pumping on the slow axis, which could be due to core ellipticity inducing a difference in the mode field diameter and thus in the nonlinear coefficient. The SC spectrum obtained when pumping at $45^{\circ}$ is narrower again because only half the power is available for spectral broadening in each axis and due to the temporal walk-off, which tends to eliminate the influence of cross-phase modulation [19]. Also, the SC spectrum is quite symmetric whatever the input angle due to the parabolic shape of the fiber dispersion. Interestingly a sideband appears at $1360 \mathrm{~nm}$, which is strongest when pumping at $45^{\circ}$ and which we identify as XPMI. When pumping on the slow axis, the sideband intensity is reduced by $12 \mathrm{~dB}$ but is still clearly observable. Finally, when pumping on the fast axis the sideband appears to be completely suppressed with an extinction of at least $25 \mathrm{~dB}$. To understand the appearance of this sideband and the efficiencies regarding the input polarization angle, let us recall that the phase matching condition for XPMI should give rise to a Stokes sideband on the slow axis and an anti-Stokes sideband on the fast axis because of positive (normal) group-velocity dispersion (GVD) [6, 20]. This means to stimulate the generation of an idler-pumped sideband in the Stokes side of the spectrum we need energy in the pump (on both axes) and energy in the anti-Stokes sideband (aligned to the fast axis). Furthermore, it is not possible to obtain an XPMI process if the beam is polarized linearly along one of the axes of the fiber as the process requires interaction between light on both axes. Experimentally, we use a pump laser with polarization extinction ratio of $40 \mathrm{~dB}$ and inject the light into the end-collapsed PM-ANDi fiber. At the output we measure a maximum achievable PER of $17 \mathrm{~dB}$, which has a relatively even distribution over the whole SC bandwidth. This shows that, at some point in the propagation, light has deviated from the input axis to become distributed more over both axes, probably due to

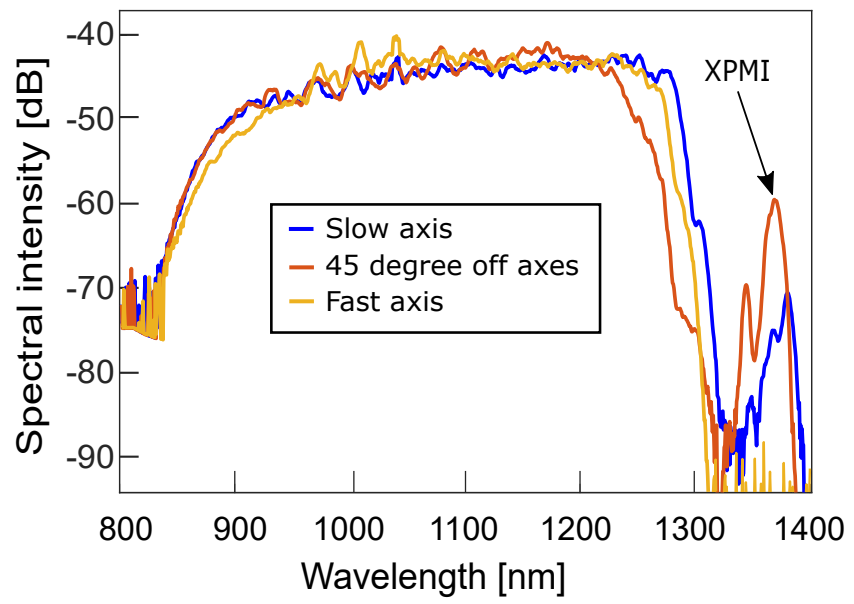

Fig. 3. Experimental SC spectra for different input polarization with $220 \mathrm{~mW}$ output power. 


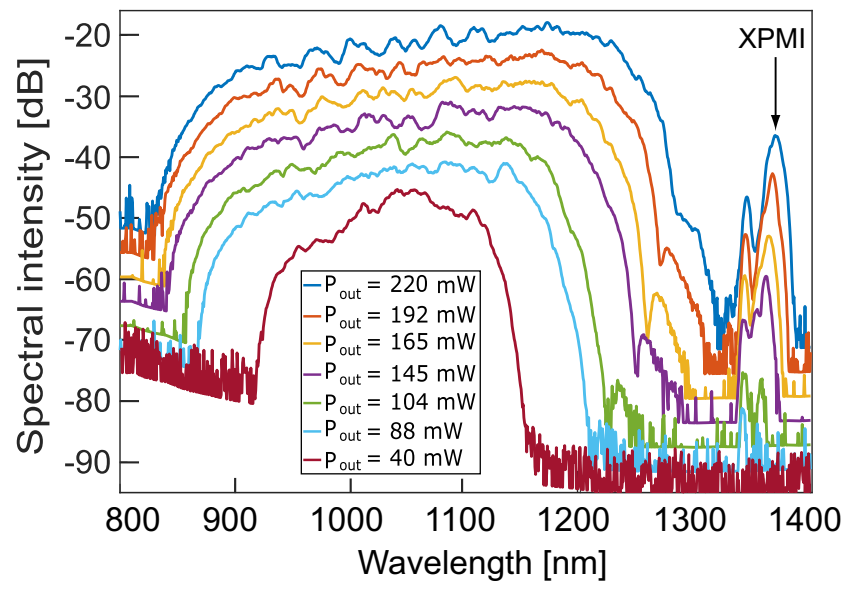

Fig. 4. SC evolution for different pump power while pumping at $45^{\circ}$ from the two axes (power offset for clarity).

the collapse of the fiber holes. This can explain why it is possible to observe a Stokes sideband at $1360 \mathrm{~nm}$ even when the input beam is almost aligned on the slow axis, though the efficiency of this sideband will be much lower than when the beam is aligned at $45^{\circ}$ of the axis, as shown in Fig. 3.

Figure 4 shows the evolution of the SC spectrum as a function of the pump power for an input polarization at $45^{\circ}$. As expected in ANDi SC generation, the broadening is limited by the dispersion of the fiber and the peak power available. The SC bandwidth is $430 \mathrm{~nm}$ at $-20 \mathrm{~dB}(845 \mathrm{~nm}-1275 \mathrm{~nm})$ for an average output power of $220 \mathrm{~mW}$. The XPMI sideband grows and slightly broadens to longer wavelengths when increasing the coupled power. It starts to appear only when SC extends past $1200 \mathrm{~nm}$ and when the OWB also starts to appear (See the green spectrum in Fig. 4). In addition, there is no observable anti-Stokes sideband outside the SC, even when observing the spectrum over a wide bandwidth (600 nm - $2000 \mathrm{~nm})$.

Adding a polarizer at the output of the fiber to analyze the sideband polarization angle, we show in Fig. 5 the SC spectrum measured after the polarizer as a function of the polarizer angle at maximum output power (220 $\mathrm{mW}$ average power). We can notice the 3 spectra obtained by aligning the polarizer on the slow axis (blue curve), fast axis (red curve) and at $45^{\circ}$ of the axes (pink curve) have a similar bandwidth and shape. We observe the most powerful sideband when the polarizer is aligned on the

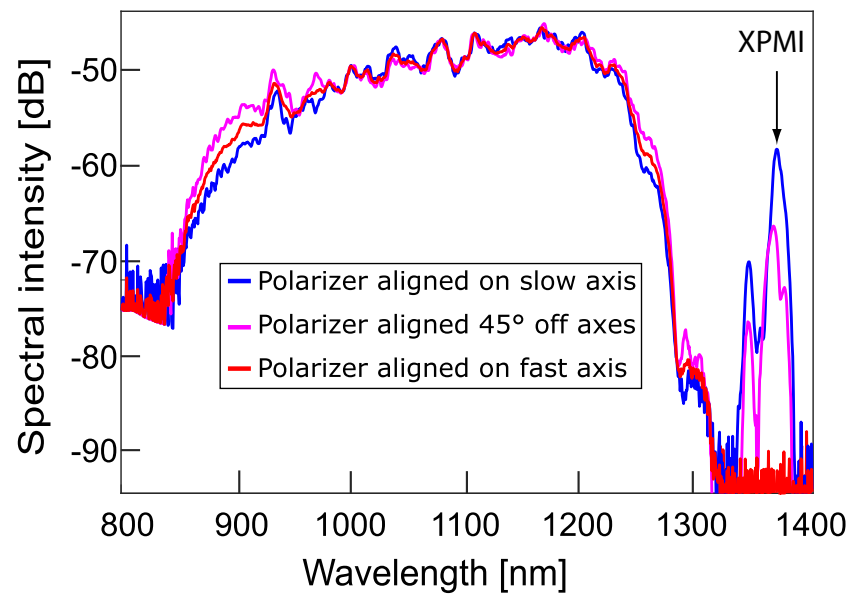

Fig. 5. SC evolution for different polarizer orientation while pumping at $45^{\circ}$ from the two axes. slow axis and a $10 \mathrm{~dB}$ suppression when aligning the polarizer at $45^{\circ}$ of the axes. Finally, aligning the polarizer on the fast axis totally suppresses the sideband, confirming that the sideband is polarized along the slow axis.
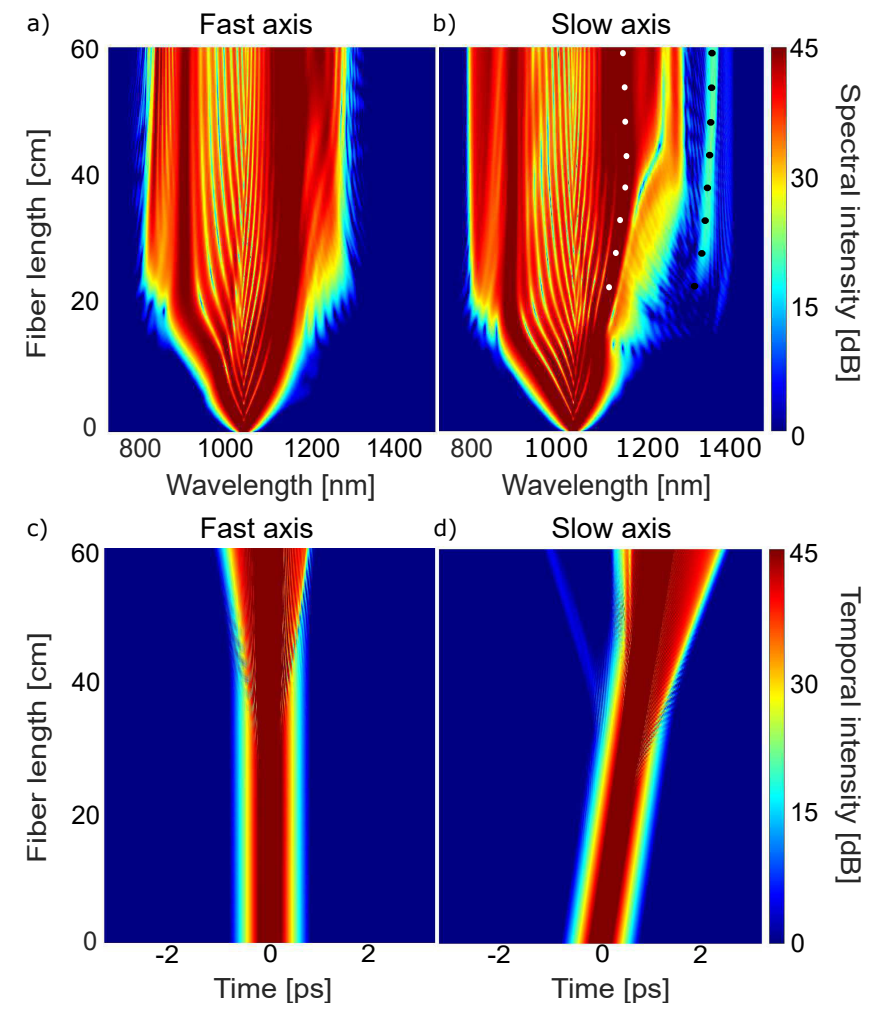

Fig. 6. Simulated spectral (top) and temporal (bottom) SC evolution on each fiber axis as a function of fiber length. The dots represent the theoretical XPMI wavelength (black) using the redder SPM wavelength (white) as a pump.

To simulate the SC generation in the PM-ANDi fiber, we use a Matlab code solving the two coupled generalized nonlinear Schrodinger equations (CGNLSE) for highly birefringent fibers as described in [21]. We used as input parameters a pump wavelength of $1040 \mathrm{~nm}$, a sech-shaped pump pulse with duration of $200 \mathrm{fs}$ (full width half maximum intensity), $12 \mathrm{~kW}$ peak power, a longer fiber length of $60 \mathrm{~cm}$ for better visibility, the loss profile as described in Fig. 2 (green curve) and the birefringence values taken from the inset. One-photon-per-mode noise and intensity noise of $1 \%$ was added to our input condition and the results were sampled average over 20 simulations. Using these parameters, we obtain a quite good agreement between simulation and experimental results. This is shown in Figs. 6(a-d) that depict both the spectral and temporal intensity dynamics on each axis when pumping the PM-ANDi fiber at $45^{\circ}$. First the numerical SC bandwidth at $-20 \mathrm{~dB}$ level is estimated to be $450 \mathrm{~nm}$ which is very close to the experimental one $(430 \mathrm{~nm})$. Second we can clearly see the generation of a small signal at $1360 \mathrm{~nm}$ polarized on the slow axis, which fits with the experimentally observed XPMI sideband (See Fig. 6b). We can also notice the sideband appears after $20 \mathrm{~cm}$ of propagation exactly where the OWB sets in and stops the red-shift of the SPM lobes. Interestingly, the temporal trace plotted in Fig. 6(d) reveals that the signal at 1360 $\mathrm{nm}$ behaves as a small dispersive wave (DW) shed by the pump pulse on the slow axis, in a way akin to the DW emission by OWB in the anomalous dispersion regime [22, 23].

To get further into details, in Fig. 7, we plot the theoretical 


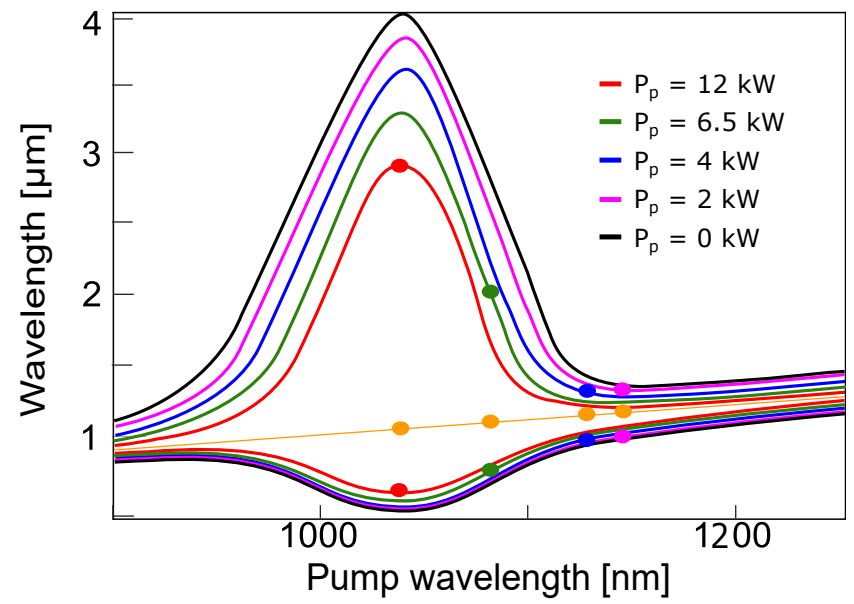

Fig. 7. Phase-matching map as a function of total peak power, $12 \mathrm{kw}$ (red), $6.5 \mathrm{~kW}$ (green), $4 \mathrm{~kW}$ (blue), $2 \mathrm{~kW}$ (pink), 0 (black). The orange dots on the orange straight line for the pump wavelength show the numerically observed red SPM wavelength (white dots in Fig. 6b) and the other colored dots mark the corresponding XPMI wavelength.

XPMI sidebands as a function of pump wavelength using the well-known formula for the XPMI frequency shift [24],

$\Omega_{S}(\lambda, z)=\frac{\delta n(\lambda)}{2 c \beta_{2}(\lambda)}\left(1+\sqrt{1-4 \beta_{2}(\lambda) \gamma P_{0}(z)\left(\frac{c}{\delta n(\lambda)}\right)^{2}}\right)$,

where $\delta n$ is the group birefringence, $P_{0}$ the total peak power, and $\beta_{2}$ the the wavelength dependent GVD. The XPMI wavelengths are plotted in Fig. 7 for different peak powers of $12 \mathrm{~kW}, 6.5 \mathrm{~kW}$, $4 \mathrm{~kW}, 2 \mathrm{~kW}$ and 0 . From the modelling, the slow axis XPMI generation is seen to be closely linked to the long wavelength SPM lobe in the slow axis, marked with white dots. According to this conjecture, the XPMI gain is only efficient when the red shift of the SPM lobe, acting as the pump, slows down, and for lengths not much longer than the walk-off length of $22 \mathrm{~cm}$. This is exactly what is observed: the XPMI peak is first growing after about $20 \mathrm{~cm}$ when the red-shift of the SPM lobe is stopped by $\mathrm{OWB}$, and after about $30 \mathrm{~cm}$ the power in the XPMI peak does not grow anymore. Looking in more detail we find that the peak power decreases from $12 \mathrm{~kW}$ at $\mathrm{z}=0$ to $1.5 \mathrm{~kW}$ at $\mathrm{z}=60$ $\mathrm{cm}$. From the corresponding phase-matching curves in Fig. 7 we see that the XPMI phase-matched to the center wavelength of the SPM lobe initially at $2787 \mathrm{~nm}\left(P_{0}=12 \mathrm{~kW}, \lambda_{S P M}=1040 \mathrm{~nm}\right)$, then it rapidly decreases because of the red-shift of the SPM lobe. The observed final SPM lobe wavelength of $1152 \mathrm{~nm}$ is seen to generate XPMI at $1360 \mathrm{~nm}$ in the valley of the linear phasematching curve, which nicely corresponds to the numerically and experimentally observed XPMI wavelength, for the adjusted dispersion profile. The observed SCG induced XPMI generation thus requires a delicate balance between strong SPM stopped sufficiently before say twice the walk-off length by OWB.

In conclusion, we have reported the observation of crossphase modulation instability while pumping a PM ANDi PCF with a femtosecond mode-locked laser. A sideband was generated through a XPMI process at $1360 \mathrm{~nm}$ during coherent supercontinuum generation from self-phase modulation and optical wave breaking. We demonstrated this sideband cannot be generated while pumping on the fast axis and is itself polarized along the fiber's slow axis. Further, clarifying that we were observing XPMI, theoretical calculation and simulation performed solving the CGNLSE confirmed the degenerate four wave mixing between a pump corresponding to the red edge of the SPM on the fiber's slow axis, an anti-Stokes idler pump in the central SPM area on the fiber fast's axis and a signal at $1360 \mathrm{~nm}$ polarized on the fiber's slow axis. Our study is of substantial value to potential applications, such as OCT and metrology, which require ultra low-noise SC light sources. To achieve low-noise in these future SC sources, a high degree of suppression of XPMI will be required and for this a solid understanding of the underpinning physics. Indeed, this study shows that control the input polarization is very important to avoid noise amplification effect and thus keep the noise-free/stability given by a fs PM-ANDi SC generation.

Funding. Horizon 2020 Framework Programme (H2020) Marie Curie grant No. (722380 [SUPUVIR]); Agence Nationale de la Recherche (ANR) (ANR-15-IDEX-0003, ANR-17-EURE-0002).

\section{REFERENCES}

1. A. Hartung, A. M. Heidt, and H. Bartelt, Opt. Express 19, 7742 (2011).

2. A. M. Heidt, J. S. Feehan, J. H. V. Price, and T. Feurer, J. Opt. Soc. Am. B 34, 764 (2017).

3. I. B. Gonzalo, R. D. Engelsholm, M. P. Sørensen, and O. Bang, Sci. reports 8, 6579 (2018).

4. E. Genier, P. Bowen, T. Sylvestre, J. M. Dudley, P. Moselund, and O. Bang, JOSA B 36, A161 (2019).

5. C. Finot, B. Kibler, L. Provost, and S. Wabnitz, JOSA B 25, 1938 (2008).

6. J. M. Dudley, G. Genty, and S. Coen, Rev. modern physics 78, 1135 (2006).

7. U. Møller and O. Bang, Electron. Lett. 49, 63 (2013).

8. A. Loredo-Trejo, Y. López-Diéguez, L. Velázquez-Ibarra, A. Díez, E. Silvestre, J. M. Estudillo-Ayala, and M. V. Andrés, IEEE Photonics J. 11, 1 (2019).

9. M. Maria, I. B. Gonzalo, T. Feuchter, M. Denninger, P. M. Moselund, L. Leick, O. Bang, and A. Podoleanu, Opt. letters 42, 4744 (2017).

10. M. K. Dasa, C. Markos, M. Maria, C. R. Petersen, P. M. Moselund, and O. Bang, Biomed. optics express 9, 1762 (2018).

11. C. R. Petersen, N. Prtljaga, M. Farries, J. Ward, B. Napier, G. R. Lloyd, J. Nallala, N. Stone, and O. Bang, Opt. letters 43, 999 (2018).

12. N. M. Israelsen, M. Maria, M. Mogensen, S. Bojesen, M. Jensen, M. Haedersdal, A. Podoleanu, and O. Bang, Biomed. optics express 9 , 2240 (2018).

13. B. Povazay, K. Bizheva, A. Unterhuber, B. Hermann, H. Sattmann, A. Fercher, W. Drexler, A. Apolonski, W. Wadsworth, J. Knight et al., Opt. letters 27, 1800 (2002).

14. K. Tarnowski, T. Martynkien, P. Mergo, K. Poturaj, A. Anuszkiewicz, P. Béjot, F. Billard, O. Faucher, B. Kibler, and W. Urbanczyk, Opt. express 25, 27452 (2017).

15. K. Tarnowski, T. Martynkien, P. Mergo, J. Sotor, and G. Soboń, Sci. reports 9, 1 (2019).

16. A. Kudlinski, A. Bendahmane, D. Labat, S. Virally, R. Murray, E. Kelleher, and A. Mussot, Opt. express 21, 8437 (2013).

17. P. Drummond, T. Kennedy, J. Dudley, R. Leonhardt, and J. Harvey, Opt. communications 78, 137 (1990).

18. P. Hlubina, D. Ciprian, and M. Kadulová, Meas. Sci. Technol. 20, 025301 (2008).

19. T. Sylvestre, H. Maillotte, E. Lantz, and D. Gindre, J. Nonlinear Opt. Phys. \& Mater. 6, 313 (1997).

20. J. Chen, G. Wong, S. Murdoch, R. Kruhlak, R. Leonhardt, J. Harvey, N. Joly, and J. Knight, Opt. letters 31, 873 (2006).

21. A. N. Ghosh, M. Meneghetti, C. R. Petersen, O. Bang, L. Brilland, S. Venck, J. Troles, J. M. Dudley, and T. Sylvestre, J. Physics: Photonics 1, 044003 (2019).

22. M. Conforti and S. Trillo, Opt. Lett. 38, 3815 (2013).

23. K. E. Webb, Y. Q. Xu, M. Erkintalo, and S. G. Murdoch, Opt. Lett. 38, 151 (2013).

24. G. P. Agrawal, in Nonlinear fiber Optics, (Springer, 2000), pp. 195-211. 